\title{
Visible-light laser diodes and superluminescent diodes: characteristics and applications
}

Omar Alkhazragi*, Jorge A. Holguín-Lerma*, Tien Khee Ng, and Boon S. Ooi

Photonics Laboratory, Computer, Electrical and Mathematical Sciences and Engineering (CEMSE) Division

King Abdullah University of Science and Technology (KAUST), Thuwal 23955-6900, Saudi Arabia

Equal contribution

E-mail:boon.ooi@kaust.edu.sa

\begin{abstract}
Semiconductor light sources technology has seen tremendous strides in recent decades and a rapidly increasing interest in it. The unique advantages and characteristics of this form of light generation include compactness, high efficiency, and reliability. With these recent advancements, light-emitting diodes (LEDs), laser diodes, and superluminescent diodes (SLDs) have become an indispensable part of our homes, factories, and research facilities. In particular, the sensitivity of the human eye to the visible range of the electromagnetic spectrum ranging from 400 to $700 \mathrm{~nm}$ and the wavelength-dependence of optical characteristics of materials make visible light required for a plethora of applications ranging from displays for entertainment, to imaging in the medical field, to light-based atomic clocks. While LEDs are the most commonly found type of semiconductor light sources, laser diodes and SLDs are of special interest due to their higher output optical power, spectral purity, and coherence. In this tutorial, we first go over the main unique characteristics of the different types and configurations of visible-light laser diodes and SLDs and their general structures with a focus on their advantages compared to LEDs. We then discuss the applications in which these characteristics are of great interest in the fields of displays, communication, instrumentation, and photonic integrated circuits.
\end{abstract}

\section{Keywords}

Laser diode, superluminescent diode, visible light, applications

\section{Introduction 2 \\ 2. Device Structure 4 \\ 2.1 Laser diode 4 \\ 2.2 Superluminescent diode 6 \\ 3. Applications 7}

3.1 Displays, augmented and virtual reality 7

3.2 Underwater optical wireless communication Error! Bookmark not defined.

3.3 Instrumentation: industry and research 10

4. Conclusions and perspective 12

References 13 


\section{Introduction}

Visible light belongs to the electromagnetic spectrum with wavelengths ranging from $\sim 400$ $\mathrm{nm}$ (violet) and $\sim 450 \mathrm{~nm}$ (blue), through $\sim 550$ $\mathrm{nm}$ (green) and $\sim 650 \mathrm{~nm}$ (red), reaching up to $\sim 700 \mathrm{~nm}$ (far red). Plants capturing sunlight, oxygen blood sensors based on a red light, and urban illumination from post lamps to displays, are examples where visible light plays a critical role. Either as broadband white light or as an individual color, visible light represents one of the most essential natural and technological resources. Consequently, the devices dealing with emission of visible light are valuable for all human, commercial, and technological perspectives.

Light sources have evolved from candles to the light bulb and further transitioned into solidstate lighting, i.e., illumination based on semiconductor diodes [1, 2]. State-of-the-art semiconductor devices based on the group-III nitride, i.e., AllnGaN/GaN or GaN-based, cover the color emission from violet to green; while the devices based on the group III-phosphide, i.e., AllnGaP/GaAs or GaP-based, cover the color range from amber to red. The realization of the first visible light-emitting diode (LED) followed early developments of near-infrared diode technology in the 1960s [3-5]. Some of the earliest computers used GaP-based redand amber-emitting LEDs as light indicators, but these pioneering LEDs were significantly expensive due to the low volume production and technological challenges of that time. At present, both GaN-based and GaP-based LEDs are manufactured in the billions and found in mobile phone displays, streetlights, vehicles, and even indoor horticulture, with GaN-based LEDs being the major player in white-light illumination.

The invention of the LED did not stop researchers and industry to pursue the realization of a semiconductor laser diode. In fact, much of the semiconductor development carried in the 1960s aimed at replicating the ruby laser [6] in a diode form. This second revolution in light-emitting devices led to the demonstration of visible laser diodes [3, 7], and in consequence, the demonstration of visible superluminescent diodes (SLD, also referred to as SLED) [8, 9].

The light of LEDs is generated and emitted in random directions making it difficult to use in projectors, photonic integrated circuits (PICs), and optical waveguides such as optical fibers. Moreover, LED light is incoherent, making it unsuitable for delivering the certainty required in optical clocks and frequency standards. In this regard, the light generated by laser diodes and SLDs provides a tool for applications where standard LEDs fall short. The present work aims at introducing the reader to the applications of lasers and SLDs emitting visible light, differentiating the optical properties of these devices against the standard LED, and highlighting their applicability to modern technology.

Laser diodes and SLDs are intrinsically a diode emitting light; hence, their structure and function are built on top of the fundamental properties of conventional LEDs. A modern semiconductor LED is based on the double heterostructure (DH) design [10], where a thin active region -normally several nanometers- is epitaxially grown in between positively-doped and negatively-doped layers, also known as pand $n$-cladding layers. The thin active region is called the quantum well, which is made thinner than the de Broglie wavelength of the electrons, usually just a few nanometers; otherwise, the $\mathrm{DH}$ is considered bulk [10]. The quantum well $\mathrm{DH}$ is the standard for modern diode light emitters. By applying electrical current in the diode, the injected electron and hole (i.e., the carrier pair) recombine at the active region, creating light with energy roughly equal to the bandgap of the semiconductor; this is $\sim 3.1 \mathrm{eV}$ for violet light (400 nm) and $\sim 1.77 \mathrm{eV}$ for far-red light $(700 \mathrm{~nm})$, at the boundaries of the visible spectrum.

The light generated in the quantum well is a product of what is known as spontaneous emission, where the carrier pairs recombine spontaneously to release their potential energy in the form of photons, i.e., the light quanta. The spontaneous emission is non-coherent and is emitted in random directions because the LED structure does not provide any significant form of optical feedback or guiding. The reader may refer to [11] for a comprehensive description of the LED phenomena and its principles of operation.

A modified LED epitaxial structure involves the integration of optical waveguide layers with a refractive index lower than that of the active region. The waveguide layers are found in the vicinity of the quantum well and are known as the separate confinement heterostructure $(\mathrm{SCH})$. In general terms, these layers provide sufficient refractive index contrast to achieve inplane optical guiding of the light. This optical waveguide is the most common feature found in edge-emitting lasers and SLDs, and it is one 
of the key features to achieve their effective operation. Nonetheless, the optical confinement given by the $\mathrm{SCH}$ is only in the vertical direction. Thus, in order to have a higher control on the guiding of light along the device, laser diodes and SLDs normally have a lateral control of the optical mode. This lateral confinement is achieved by index contrast or gain guiding, normally in the form of a ridge waveguide.

Another type of high-performance laser diode is the vertical-cavity surface-emitting laser (VCSEL). For VCSELs, the light emission is out-of-plane, and unlike edge-emitting devices, $\mathrm{SCH}$ layers are not present. Nonetheless, optical mode confinement is achieved by an aperture, which is regularly an oxide isolation layer in the form of a ring, providing both optical and electrical confinement and guiding in the vertical direction [12]. The oxide aperture can also be relieved using selective etching to improve the high-speed performance of the VCSEL $[13,14]$. Summarizing, the light coming out of a laser (both edge-emitting and VCSELs) or SLD is a guided light beam; this results in their spatial coherent. The recent progress of GaN-based visible-light VCSELs has been reviewed in Ref. [15].

For lasers and SLDs, at first, spontaneous emission appears in the quantum well too. However, some portion of this light is waveguided along the quantum well by the $\mathrm{SCH}$ and the lateral mode confinement; or the aperture in VCSELs. By increasing the injection of electrical current, the growing number of carriers will achieve a condition known as population inversion, where the majority of electrons and holes are in an excited state. At this moment, the photons coming through the waveguide will induce the generation of stimulated light by photon-carrier interaction and carrier pair recombination in the active region at a rate greater than the absorption rate or the optical losses in the device. This light stimulation reaction happens multiple times along the waveguide. The newly generated photons will then share the same direction, phase, polarization, and wavelength of the initially incoming photons creating a wave of temporally and spatially coherent light, known as stimulated emission. Nonetheless, the process of light amplification can be undermined by optical absorption or losses withing the quantum well, hence, an optical feedback is necessary to sustain laser light, which increases the time spent by photons inside the active region, allowing them to stimulate even more emission of identical photons, increasing the coherence.

The optical feedback driving the amplification process is provided by optical mirrors found in both edge-emitting lasers and VCSELs. These mirrors will make light travel back and forth within the laser cavity. In this way, the mirrors form the laser cavity, also known as a FabryPérot resonator, supporting the continuous generation of stimulated emission and highly coherent laser light. At this point, the coherence of light, both in time and space, is further increased.

Unlike laser diodes, SLDs do not have a cavity but retain the optical waveguide of the laser design and a high-reflective mirror on one side of the device. Thus, SLDs are referred to as double-pass devices. Given the lack of a cavity in SLDs, the stimulated emission is less promoted creating an intermediate regime called amplified spontaneous emission (ASE) where high temporal coherency is not achieved despite being operated under the stimulated emission amplification process. This is due to the reduced amount of time a photon spends inside the gain medium, decreasing the amount of emission it can stimulate, which allows more electrons to emit light spontaneously.

Near-infrared (NIR) SLDs have garnered significant attention since their introduction in 1971 due to their advantages in applications that require short coherence lengths [16]. However, III-nitride SLDs, which were demonstrated for the first time in 2009 [8], opened the door for these applications to make use of shorter wavelengths ranging from visible light to ultraviolet. This wavelength range is important for visible-light applications (displays and illumination) and for spectroscopy (using external cavities). SLDs can also be used with external cavities in laser cooling and atomic clocks, which require specific wavelengths. The shorter wavelength also results in a smaller diffraction limit.

The differences in light amplification between visible LED, SLD, and laser diode will affect the properties of the light output. The color purity related to the optical bandwidth of the light emission will vary accordingly, LED being the device with the wider optical broadening. On the other hand, the stimulated emission and the optical feedback of the laser diode will make this device approach monochromatic emission, depicting a narrow optical bandwidth. SLDs represent an intermediate stage between the broad LED emission spectrum and the narrow 
linewidth of laser light, thus complementing a set of devices with unique properties deemed necessary for a variety of applications. The visual appearance of light produced by GaNbased blue laser, SLD, and LED is shown in Figure 1. The increasing speckle density in laser light, as compared to SLD and LED light is due to the higher degree of coherence, leading to interference artifacts.
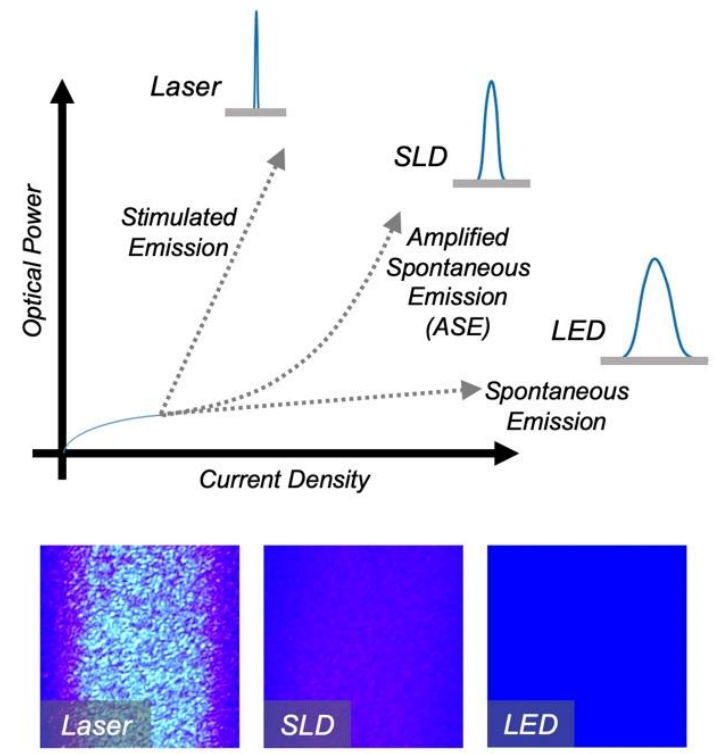

Visual appearance

Figure 1: Upper: The relative relation of output power and optical bandwidth produced by laser diodes, SLDs, and LEDs. Bottom: Corresponding visual appearance of the light generated by a GaN-based blue laser diode, SLD, and LED [17].

The optical narrowing of the amplification process will be accompanied by optical power behavior. LEDs are generally described by a linear relation of optical output power against injected current. Laser diodes also follow a linear relation but with a higher positive slope after a lasing threshold where stimulated emission becomes fully dominant. SLDs are characterized by a gradual exponential increase due to the ASE. Figure 1 shows a representation of optical bandwidth and optical power comparing LEDs, SLDs, and laser diodes.

\section{Device Structure}

\subsection{Laser diodes}

The classical design of an in-plane Fabry-Perot laser diode structure is shown in Figure 2(a). The epitaxial structure is grown on a native substrate. Such native substrate ( $\mathrm{GaN}$ for blue and green lasers, for example) has a crystal lattice constant comparable to most of the top epitaxial layers in order to reduce crystal defects and improve the device efficiency. Other substrates with highly mismatched lattice constant and crystal structure (sapphire) can also be used but this can lead to an increased number of defects and therefore compromise the material quality; this is usually a critical consideration for laser diodes. Nevertheless, mass-production substrates such as silicon remain attractive for large scale integration, and thus, buffer layers and strain compensating methods have been developed to grow visiblelight laser structures on such platforms [18].

On top of the substrate, the $n$-cladding layer is grown ( $n-G a N$, for example), followed by the undoped active region (InGaN/GaN quantum wells/barriers for blue and green and AIGalnP/GalnP for red) and the upper $p$ cladding layer ( $p-G a N$, for example). This process will form the $\mathrm{SCH}$ and the quantum well. The doping level of the cladding layers is regularly in the range of $10^{17}-10^{18} \mathrm{~cm}^{-3}$. Semiconductor contact layers with high carrier concentration are needed to create the interface between the semiconductor diode and the metal contacts. A high carrier concentration in the order of $10^{18}-10^{19} \mathrm{~cm}^{-3}$ is needed for the contact layers. Achieving such high doping concentration is challenging for the case of $p$ type $\mathrm{GaN}$, and hence, besides the conventional doping methods using foreign impurities, polarization-based carriers concentration enhancement [19] has also been developed to circumvent some of the challenges.

The epitaxial layers of a laser diode vary based on the target wavelength. As an example, a blue laser diode epitaxial structure can consist of a 1-3 $\mu \mathrm{m}$ n-type GaN cladding layer, a 100$\mathrm{nm} n$-type $\ln _{\times 1} \mathrm{Ga}_{1-x 1} N$ waveguide layer (forming $\mathrm{SCH}), \quad \quad \mathrm{In}_{\times 2} \mathrm{Ga}_{1-\times 2} \mathrm{~N} / \mathrm{In}_{\times 1} \mathrm{Ga}_{1-\times 1} \mathrm{~N}$ quantum well/quantum barrier pairs (where $\mathrm{x}_{2}>\mathrm{x}_{1}$ ) forming the undoped active region, a p-type $100-n m \ln _{\times 1} G_{a_{1-x 1}} N$ waveguide, and a 100-nm $\mathrm{p}^{++}-\mathrm{GaN}$ contact layer. Due to $\mathrm{p}$-type doping challenges, the $p$-type contact layer can be split into a thick moderately doped layer capped by a thin highly doped $\mathrm{p}^{++}$-type layer. An AIGaN electron-blocking layer can be added on top of the active region.

The quantum well $\mathrm{DH}$ design plays an important role in laser diodes. The heterojunction barriers prevent injected carriers from diffusing to adjacent layers, which results in a thinner active region, i.e. the quantum well. Therefore, the population inversion condition can be achieved at a lower current density, 


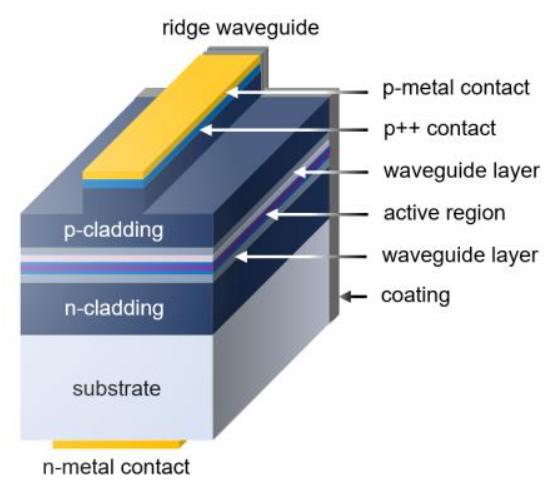

(a)

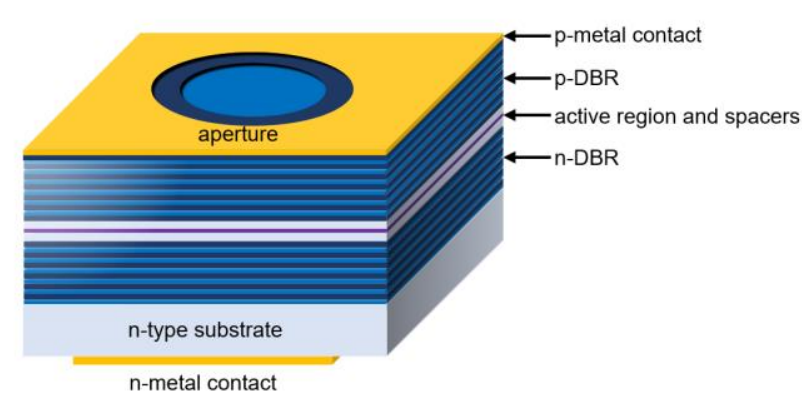

(b)

Figure 2: General design of (a) an edge-emitting laser diode (or SLD) and (b) a vertical-cavity surface-emitting laser (VCSEL).

improving the laser threshold and the general performance of the devices. Moreover, the high refractive index of the active region improves the optical confinement, which increases the overall gain. The high bandgap of the top and bottom layers prevents them from absorbing the emitted photons, reducing the optical losses and improving the efficiency of the laser diode. Such a single-quantum-well (SQW) laser can achieve a narrow spectral linewidth and a low threshold current. A multi-quantum-well (MQW) structure, generally formed by two or three quantum wells, can be used to improve the gain at higher injected current densities, but a broadening in the spectral linewidth is expected.

After the epitaxial growth, the wafer should be processed in cleanrooms to create the laser chips. This process involves photolithography, etching, insulator and metal depositions, as well as annealing processes. As mentioned before, the key features for the laser diode include creating a longitudinal waveguide. In Figure 2(a), the waveguide is depicted as a ridge, which is one of the most commonly used methods. Other waveguiding methods include gain guiding by electrical injection, index guiding by proton implantation or porosity index change, and buried waveguides. Details about lateral optical confinement (waveguiding) can be found in Chapter 1 in Ref. [12]. In all cases, the laser diode is consolidated by further cleaving or etching the facets in order to form the laser cavity. The resulting laser facets provide a non-negligible reflectivity due to the air-semiconductor interface, which is enough for achieving lasing, but not in every case. Thus, high-reflection (HR) coatings with reflectivity around $95 \%$ are normally deposited on the back facet. Laser devices dealing with high optical powers would require a front facet section or coating made of a material with a large bandgap, i.e., larger than the laser emission. This will help to avoid catastrophic optical damage (COD) at the output facet. However, it is worth mentioning that $\mathrm{GaN}$-based lasers have higher rigidity and thermal conductivity compared to AlGaAs-based lasers, which makes their COD threshold optical density substantially higher (tens of $\mathrm{MW} / \mathrm{cm}^{2}$ compared to a few $\left.\mathrm{MW} / \mathrm{cm}^{2}\right)$ [20-22]. The mirror loss $\left(\alpha_{m}\right)$ of a laser resonator is inversely related to the reflectivity of the facet mirrors ( $R 1$ and $R 2)$ and the laser cavity length $(L)$ as $\alpha_{m}=\frac{1}{2 L} \ln \left(\frac{1}{R 1 R 2}\right)$. Thus, HR coating technology is valuable for visible laser diodes. Similarly, the length of a laser diode has a significant impact in the device properties, and it is usually found to be in the order of several hundreds of micrometers.

Together with the device length, the ridge or gain width have an impact in the electro-optical properties of the laser diode. These dimensions will influence the injection area and, more importantly, will also determine the lateral mode confinement, leading to either a singletransverse-mode or a multi-mode laser. Ridge widths, ranging from a couple of micrometers in the narrow-ridge lasers, to more than ten micrometers in the broad area (BA) lasers, will influence the mode selectivity. Other factors in laser diode design involve the optical gain, scattering loss, and the optical confinement factor, which are related to the material properties, composition, and wavelength of operation, with further reading available in references [12, 23].

A working example of commercial lasers can be chosen from any of the leading visible laser diode manufacturers such as Nichia, Osram, Mitsubishi, Sony, Ushio, and others. These companies show a track record of using native 
substrates, ridge waveguide self-aligning techniques, and high-bandgap front facet coatings and treatments to avoid COD on wattlevel visible lasers, as well as lasers of lengths up to approximately $1 \mathrm{~mm}$. Of course, proprietary epitaxial structures are less known to the literature.

For the case of VCSELs, the classical design is shown in Figure 2(b). The semiconductor epitaxy follows the same material considerations and challenges of the edgeemitting laser, with additional complexity given by the need of integrating the bottom and top mirrors, as well as the isolation layer to form the laser aperture for electrical and optical confinement. The efforts behind the VCSEL deserve stand-alone studies such as previously reported in [24]. The realization of visible VCSELS required several years of additional development following the demonstration of its edge-emitting counterpart [25, 26]. Several challenges involved the overall optical and carrier lateral confinement design, including conductive layers for lateral injection of electrical current, prevention of p-layer absorption, as well as the realization of highreflectivity mirrors with greater than $99 \%$ reflectivity, necessary due to the extremely short cavity length. Nevertheless, VCSELs stood out due to their many attractive features such as single longitudinal mode operation, remarkable beam quality, on-wafer testing and high-volume manufacturability, as well as twodimensional array integration.

The single longitudinal mode operation of VCSELs is due to the short cavity of the device providing a large free-spectral range (FSR), following the relation $F S R=\frac{c}{2 n_{\mathrm{eff}, \mathrm{g}} L}$, or the equivalent mode spacing $(\Delta \lambda)$, following the relation $\Delta \lambda \approx \frac{\lambda^{2}}{2 n_{\mathrm{eff}, \mathrm{g}} L}$, where $n_{\mathrm{eff}, \mathrm{g}}$ is the group effective refractive index of the cavity, $c$ is the speed of light in free space, $\lambda$ is the wavelength of the laser in free space, and $L$ the cavity length.

To achieve single longitudinal mode operation in edge-emitting laser diodes, wavelengthselective gratings are needed. The FSR in the relatively long cavities of edge-emitting lasers is much smaller than the FSR of VCSELS, thus, allowing multiple adjacent cavity longitudinal modes to coexist in a practical laser diode. Features such as the distributed-feedback (DFB) laser utilizes a Bragg grating with alternating refractive indices to select a single wavelength for lasing according to the Bragg condition. A similar design relies on two DBR gratings on both ends of the laser functioning as wavelength selective mirrors.

Overall, the light of a laser diode provides a seemingly small linewidth emission, especially when compared against LEDs and SLDs, but despite this fact, the differences in linewidth between a standard Fabry-Pérot laser diode and a single-longitudinal-mode laser diode are significant for certain applications. The optical linewidth of diode-based light emitters will eventually become a key element on their usability and value, and thus, forms the base of further discussion in this document.

\subsection{Superluminescent diodes}

The design of a visible-light superluminescent diode (SLD) follows exactly the same epitaxial growth (InGaN for blue and green, and AIGalnP for red) and fabrication steps needed for edgeemitting laser diodes [Figure 2(a)], including the lateral confinement structures such as the ridge waveguide. However, it is necessary to suppress the formation of the resonant longitudinal modes through at least one of a variety of approaches, including tilted facets, integrated absorbers, anti-reflective coatings, curved ridge waveguides, and etched facets. In any of these approaches, the single-pass optical gain $\left(G_{s}\right)$ is an important parameter. This is similar to the semiconductor optical amplifier (SOA), with the difference being that SLD amplifies its own spontaneously generated light. In SLDs where one of the facets has a high reflectivity, i.e., double-pass SLDs, the optical power is proportional to the square of the single-pass optical gain. The single-pass optical gain is given by $G_{s}=\exp \left[\left(\Gamma g_{0} \eta \frac{J}{d}-\right.\right.$ $\alpha) L]$, where $\Gamma$ is the optical confinement factor, $g_{0}$ is the gain coefficient of the active region, $\eta$ is the quantum efficiency, $J$ is the applied current density, $d$ is the thickness of the active region, $\alpha$ is the absorption coefficient of the material, and $L$ is the length of the device. The gain and absorption coefficients, as well as the quantum efficiency, are all affected by the material quality and properties of the semiconductor used to fabricate the device. In this sense, they can only be improved or modified by epitaxy. The confinement factor and active region thickness are also defined at the time of fabrication and are the result of epitaxial and optical design. Similar to the laser diode, the chip length is a parameter with a significant impact on the optical properties of the device while at the same time being easy to modify. Increased lengths lead to higher light 
amplification due to higher optical gain but can also lead to early optical gain saturation. Detailed advances in III-nitride-based SLDs can be found in Refs. [27, 28].

Similar to edge-emitting laser diodes, the configuration of conventional SLDs designed to emit light through their edge limits on-wafer testing and makes the manufacturing of SLD arrays challenging. Surface-emitting designs are more suitable for array formation. Despite the fact that surface emission designs can be easily achieved in LEDs and laser diodes, similar designs cannot be easily adopted for SLDs, mainly because of the thin active region. The small active-region thickness does not provide enough double-pass gain. Therefore, ASE does not take place. While VCSEL designs resolve this issue by increasing the reflectivity of the cavity mirrors to ensure multiple passes of the photons through the device gain medium, optical feedback can result in lasing action, which makes it unsuitable for SLD designs.

However, apparent vertical emission from SLDs has been reported using different designs. Grating out-couplers and micro mirrors used to redirect the emitted photon through the surface have been proposed [29] [30]. It is also possible to fabricate a sloped waveguide to achieve surface emission by relying on the total internal reflection of the device [31]. However, it is important to note that these surface-emission designs that rely on the horizontal waveguide design introduce optical losses or limit the highvolume manufacturability of SLD arrays.

\section{Applications}

The characteristics of visible light such as wavelength size, photon energy, or color rendering characteristics, make the visible light emitted by laser diodes and SLDs attractive for applications discussed as follows.

\subsection{Displays, augmented and virtual reality}

The relative ease of collimation, wide range of optical output power, and color consistency have made visible laser diodes stand out for applications in light shows, holographic displays, wall projectors, home TV displays, and retinal projectors. Moreover, the high spectral purity of laser diodes makes them an ideal choice for expanding the color gamut of displays and projectors [32].
To form a color display, three primary colors are needed: red, green, and blue. By controlling the intensity of each color in a single pixel, the overall observed color by the human eye can be controlled precisely. Since lasers have narrow spectral linewidths, the range of colors they can produce is substantially wider than that of LEDs and other competing display technologies.

While laser-based displays have been in development for decades, the recent advancement in laser diodes, especially in high-power green-color laser diodes based on InGaN, opened the door for low-cost, compact laser displays. Prior to the extension of the emission wavelength of $\mathrm{InGaN}$-based lasers to the $>520-\mathrm{nm}$ range, laser displays relied on second-harmonic generation (SHG) techniques to produce the needed green light. This is done by doubling the frequency of a NIR laser emitting around $1060 \mathrm{~nm}$, for example. The NIR laser can be a diode-pumped solid-state laser or a laser diode [33,34]. This technique can also be used for all three colors by doubling three different frequencies [35]. Replacing these SHG green lasers with laser diodes allows for a significantly more compact design at a lower cost. While edge-emitting lasers can be used in scanning-based projectors, forming a large array of these lasers to create a display is challenging.

On the other hand, VCSEL arrays can easily be monolithically fabricated. However, unlike their NIR counterparts, visible-light VCSELs are still immature for the aforementioned fabrication challenges. Recently, some of these challenges have been addressed by using novel DBR designs based on curved mirrors or nano-porous n-type GaN to create blue- and green-light VCSELS [36-38]. Moreover, a quantum-dot-based VCSEL design that can be used to produce laser light in the blue-green range has also been previously demonstrated [39].

The brightness (defined as the optical power divided by the product of the source area and its solid angle of divergence [40]) of laser-based displays is also improved considerably compared to LED-based displays due to the high directionality of laser light arising from its high spatial coherence. However, it is this high degree of spatial coherence that results in speckle noise from rough surfaces [41]. Compared to incoherent light sources, such as LEDs, the speckle noise degrades the image quality significantly. Reducing the degree of spatial coherence is possible and is needed to 
improve the quality of the formed images from projectors [41, 42].

Visible-light SLDs, however, have a lower degree of spatial coherence than laser diodes while maintaining higher spectral purity compared to LEDs. For these reasons, SLDbased displays combine the low speckle noise of LEDs with the high brightness and moderately wide color gamut of lasers. A compact red-green-blue SLD module for projection, as seen in Figure 4, has been recently reported [43, 44]. The choice between SLDs and laser diodes depends on the needed color gamut and light uniformity. The narrow linewidth of lasers makes them more suitable for achieving a wider color gamut while SLDs provide a lower speckle density.

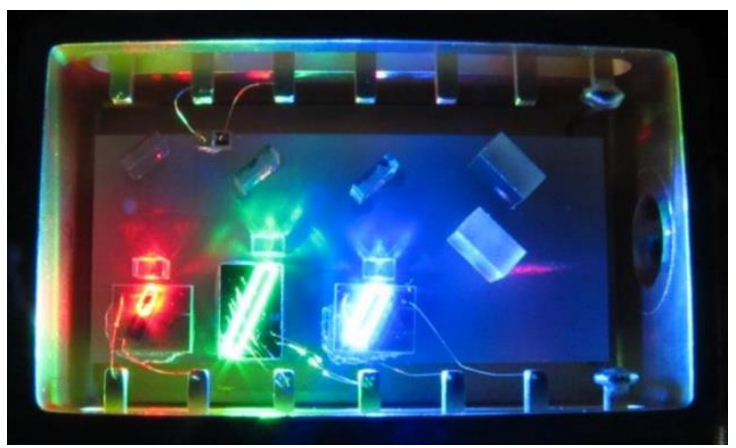

Figure 4: Photograph of a red, green, blue SLD module in operation. Reproduced with permission from [43].

Besides display panels, different types of displays can be formed using laser diodes and SLDs. Raster scanning combined with a single red-green-blue laser source can be used in laser- and SLD-based projectors. These can also be used in head-up displays and smart glasses. The scanning is done using mirrors based on microelectromechanical systems (MEMS). They can also be used in retinal projection and holographic displays [45-47]. In retinal projection, the power requirement is substantially lower since the light is projected directly to the eyes at eye-safe levels. Figure 4 shows a schematic of headset composed of the various optics needed to focus and scan laser light of the three primary colors into the retina [48]. The figure also shows the projected image by a green laser as captured by a camera.

\subsection{Underwater optical wireless communication}

Wireless communication based on visible light has attracted significant attention. By sending data using the widely available LEDs used as
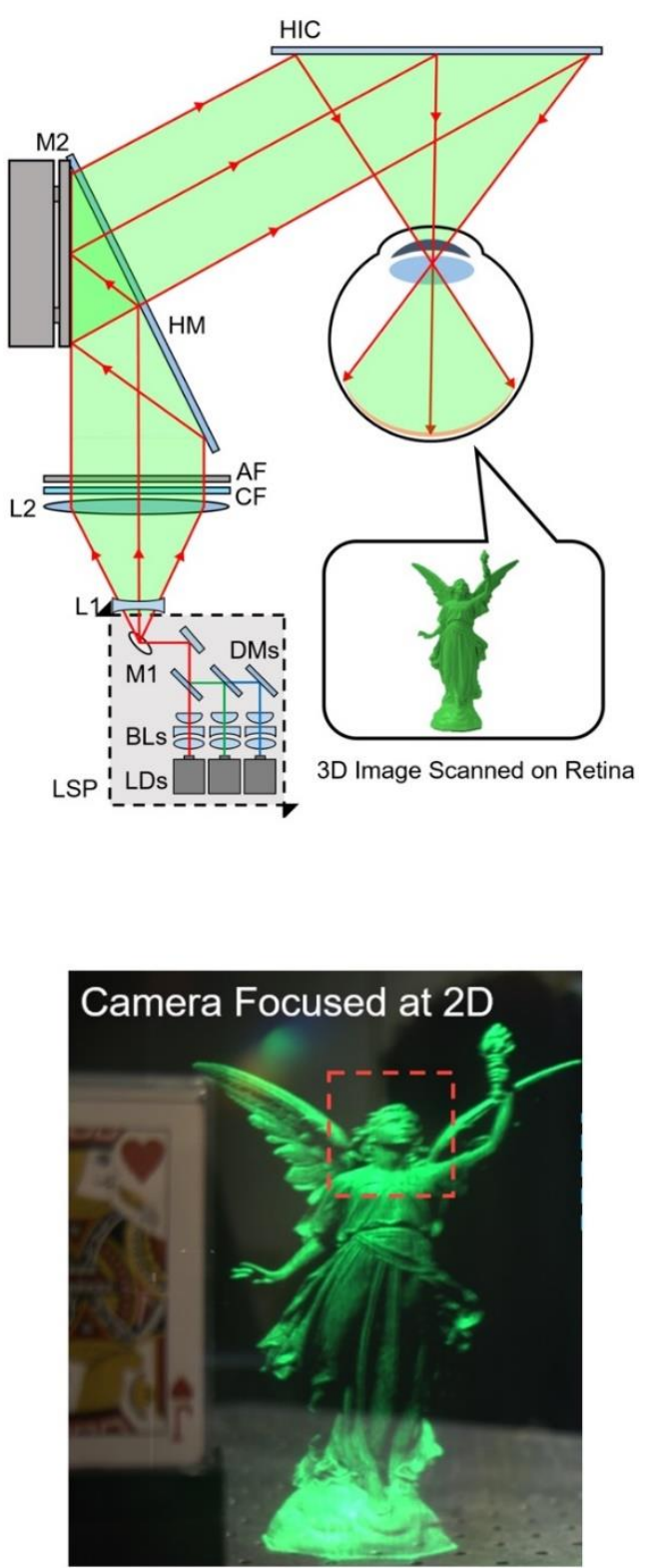

Figure 3: Schematic of a headset for retinal projection of red, green, and blue lasers. The bottom image shows a real image projected by this system. HIC: holographic image combiner, $\mathrm{HM}$ : half mirror, $\mathrm{AF}$ : attenuating filter, CF: color-balancing filter, DM: dichroic mirror, BL: beam-shaping lens, LSP, laser scanning projector, L: lens, M: mirror. Reproduced with permission from [48].

lighting sources, light-based communication can save energy and provide high-speed data transmission. However, laser diodes and SLDs have significantly broader modulation bandwidths than LEDs, and so, they can further improve the data throughput by more than an order of magnitude. SLDs in particular combine the high lighting quality of LEDs with the high modulation bandwidth approaching that of laser diodes, making them an attractive middle- 
ground solution for simultaneous high-speed communication and lighting [27]. The narrow linewidth of DFB lasers and VCSELs also allows for dense wavelength-division multiplexing (DWDM), in which separate data streams can be transmitted over the same channel using different wavelengths. Furthermore, due to their higher optical power and directionality, laser diodes and SLDs can be used in as transmitters in communication links over relatively long distances. This is especially important in underwater communication.

Traditional wireless communication technologies based on the use of radio frequency (RF) waves are impractical in underwater environments. This is due to the high attenuation experienced by electromagnetic (EM) in the RF range. The relatively high conductivity, $\sigma$, of seawater (around $4 \mathrm{~S} / \mathrm{m}$ ) causes this attenuation, especially for high frequencies. The relationship between the attenuation coefficient, $\chi(\omega)$, and the angular frequency, $\omega$, can be expressed as follows [49]:

$$
\chi(\omega)=\frac{\sqrt{8 \pi \sigma \mu \omega}}{c},
$$

where $\mu$ is the permeability of the seawater and $c$ is the speed of light. As the frequency increases, the attenuation increases, which limits the overall usable bandwidth for longrange transmission.

The limited modulation bandwidth causes the maximum achievable data rate to be orders of magnitude smaller than those usually available using RF communication in free space. Given these limitations on the propagation distance and the available bandwidth, other techniques have been developed for high-speed data transmission underwater.

The main alternative for RF in underwater environments has been acoustic communication, in which sound waves are used to transmit data. They can propagate for significantly longer distances in seawater compared to RF waves. However, acoustic communication is hindered by several factors such as noise, multipath and the high latency, the latter being due to the low speed of sound waves in water $(1.5 \mathrm{~km} / \mathrm{s})$ compared to $E M$ waves $\left(2.26 \times 10^{5} \mathrm{~km} / \mathrm{s}\right)$. This makes acoustic communication impractical in which timely response is crucial, such as in military applications. Moreover, the product of the speed, $r$, offered by acoustic communication and the transmission distance, $R$, is limited by the following upper bound [50]:

$$
R \times r \leq 40 \mathrm{~km} \cdot \mathrm{kbit} \text {. }
$$

This means that while acoustic communication can in fact support transmission for relatively long distances, the maximum data rate it provides is limited. For applications in which high data rates are required and huge amounts of data are needed to be transferred every second, such as in internet of things (IOT) networks and in live video streaming applications, a different communication technique is required that can provide a broad bandwidth and can support long-distance transmission.

The use of optical wireless communication (OWC) in the visible band is an excellent alternative to the aforementioned technologies $[51,52]$. Optical waves in the blue and green range of the EM spectrum experience minimal attenuation in seawater, allowing them to effectively propagate over relatively long distances when compared to RF waves, as can be seen in Figure 5. Moreover, with the latest developments in semiconductor light sources, compact, power-efficient transmitters featuring modulation bandwidths in the $\mathrm{GHz}$ range have become accessible and affordable. These advancements have brought about a surge in the study of underwater OWC (UOWC) systems. These systems include line-of-sight (LOS) [53, 54], diffuse-LOS [55], and non-LOS links [56]. Each of these configurations is more suitable for certain applications depending on the required coverage and the ease of alignment of the communication link. For example, Figure 5 shows the transmitter in a non-LOS link. The receiver can detect the scattered light underwater. In a LOS link, on the other hand, the receiver is placed on the opposite side facing the transmitter.

While the use of LEDs as well as laser diodes is possible, laser diodes have the advantages of having broader modulation bandwidths and higher optical power that allows for longdistance transmission. The selection of the best transmitter based on the intended channel is essential. Different factors need to be taken into consideration before establishing the communication link, including the link configuration, the inherent optical properties (IOPs) of the water in the channel, and the turbulence induced fading.

For most LOS links, laser diodes with blue or green emission are more practical given the low attenuation of light in the blue-green region of the EM spectrum. The attenuation coefficient, $c$, is the sum of the coefficients of the absorption, $a$, and the scattering, $b$. Multiple studies have been demonstrated based on blue laser diodes 
that achieved data rates beyond Gbit/s and transmission distances of around $20 \mathrm{~m}$ [53, 5759]. Green light has also been shown to be more practical in other environments that are more turbid with transmission distances up to $34.5 \mathrm{~m}[54,60]$. In highly turbid water, however, it was shown using VCSELs that the use of red light is more practical, due to the lower attenuation in these environments [61]. The use of visible-light LEDs is also possible, but they cannot offer the broad bandwidth of laser diodes [62-64]. While SLDs in underwater communication is also possible, laser diodes are preferred in any scenario in which simultaneous illumination is not needed due to their higher speed and optical power. Different techniques are used to improve the data rate and the reliability of the underwater links. High spectral efficiency modulation schemes provide significant improvements. These techniques include orbital angular momentum multiplexing [58], orthogonal frequency-division multiplexing $[54,59,65]$, wavelength-division multiplexing [66], and, more recently, probabilistic shaping (PS) [62].

Optical communication can complement the existing networks of acoustic-based underwater communication links. While acoustic communication can be established over for kilometers underwater (compared to meters for RF and tens of meters for optical OWC), they suffer from a limited modulation bandwidth $(<1 \mathrm{MHz})$ and high latency $(1.5-\mathrm{km} / \mathrm{s}$ speed compared to $2.26 \times 10^{5} \mathrm{~km} / \mathrm{s}$ for EM waves) [67]. The data rates offered by underwater OWC is unparalleled (in the range of Gbit/s) [53, 54, 60], which opens the door for applications that require high data throughput, such as live video streaming, which also benefits from the low latency. It is also worth noting that underwater OWC suffers from strict alignment requirements, unlike acoustic communication. However, the directional data transmission can be of use in applications that require a degree of privacy.

\subsection{Instrumentation: industry and research}

Laser diodes and SLDs have many attractive features that make them ideal for a wide variety of industrial and research applications. Their compact formfactor and low manufacturing cost allowed their widespread in different fields, ranging from medical imaging, to indoor horticulture, to spectroscopy. The controllability of the emission spectrum is another advantage of these devices that is critical in wavelengthsensitive applications.

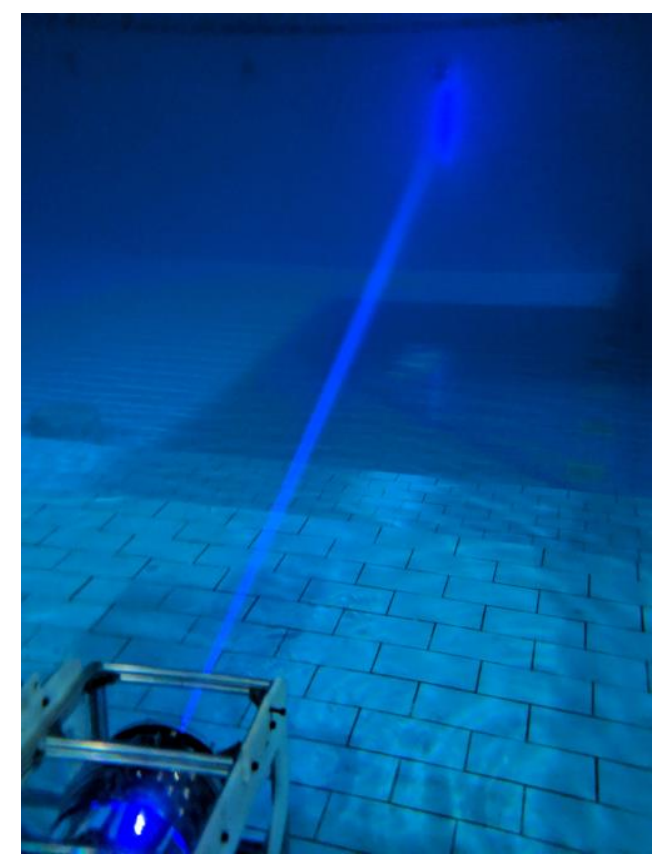

Figure 5: A photo of an underwater OWC 450-nm laser transmitter showing high power transmission $(50 \mathrm{~mW})$ over a moderately long distance $(10 \mathrm{~m})$ in a diving pool.

In spectroscopic applications, for example, tunable narrow-linewidth emission is required. To achieve that, external cavities are utilized to tune the wavelength by using a grating or changing the cavity length [68]. The longer external cavity also improves the linewidth and reduces the FSR, which improves the spectroscopic resolution of the system. The output facet of the laser diode in such a system can be antireflection coated to minimize the effects of the internal cavity whose FSR is significantly wider than that of the external cavity (transforming the laser diode into an SLD) [68]. In other words, in cases which rely on external cavities, an SLD is more suitable. However, in the absence of an external cavity, a laser diode would be required for spectroscopy due to its narrow spectral emission. Laser cooling, which also requires precise control over the emission spectrum, might also make use of external cavities and injection locking [69-72].

In Doppler cooling [73], which is used in highprecision atomic clocks, a laser beam with a precisely controlled frequency is not absorbed by the randomly moving atoms to be cooled down. This is done by ensuring that the frequency is slightly lower than that of the atoms' electronic transitions. However, due to the Doppler blueshift in the frequency for atoms moving toward the laser source, these atoms absorb the photon, reducing their overall 
momentum due to the random nature of the spontaneous emission that results from the photon absorption. In other words, the variations in the velocities of the atoms decreases, which lowers the temperature.

Another application in which the emission spectrum control is of use is indoor horticulture, which uses artificial light sources with spectra matching the absorption of the intended plants [74]. The high efficiency and directionality can also reduce the required power for these farms. Furthermore, wavefront modulation can be used in holographic systems that can redistribute the light efficiently and direct it to the plants [75].

The coherence of laser diodes and SLDs is another feature that can be utilized in various applications. These applications rely on the interference of light waves. For example, they can be used in displacement sensors [76], in optical coherence tomography (OCT), which is widely used in the medical field to study tissues non-invasively, and in fiber-optic gyroscopes (FOGs) [77].

In OCT, the light beam is split into two paths in a Michaelson interferometer using a beam splitter. The tissue to be studied is placed at the end of one of the two paths and a mirror is placed at the end of the other path. The reflected light beams from the tissue and the mirror travel back to a detector through the beam splitter. Only the light reflected from layers of the tissue within a thickness equal to the coherence length of the light source interfere with the light reflected from the mirror. The coherence length is defined as the length is space for which two points along the propagation direction are correlated and can experience interference. Therefore, having a short coherence length improves the axial resolution of the OCT system. By sweeping the position of the mirror, we can control the depth whose interference pattern is observed at the detector. It is also possible to study the spectrum of the received interference pattern to extract the information about the depth profile through a Fourier transform of the received spectrum.

Since the coherence length is inversely proportional to the linewidth of the emission spectrum of the used light source, SLDs are preferred for this application over laser diodes $[78$, 79]. Moreover, light with a shorter wavelength has a significantly shorter coherence length for the same linewidth, making visible-light SLDs ideal for OCT.
Besides the axial resolution, the scanning resolution in the transverse plane is enhanced by using shorter wavelengths due to the smaller diffraction limit (which is equal to $\lambda / 2 N A$, where $N A$ is the numerical aperture of the objective lens), which is another advantage of relying on visible light in obtaining high-resolution OCT cross-sectional images of the tissue of interest [78].

The short coherence length of visible-light SLDs is also advantageous in FOG systems, which rely on the Sagnac interference to measure the orientation. The light reflected through Rayleigh scattering in the optical fiber within a segment equal in length to the coherence length interferes with the signal, introducing noise. Therefore, SLDs are more suitable for FOGs compared to laser diodes whose coherence length is longer [80, 81].

Laser diodes in visible wavelengths, particularly in the red region (630 $\mathrm{nm}$ to $680 \mathrm{~nm})$, are used for health treatments known as low-level laser therapy [82, 83]. The exact function of laser light interacting with cells remains a topic of investigation, but with the current availability of laser diodes emitting at several wavelengths in the blue, green, and red region, low-level laser therapy continues to attract attention.

The integration of visible lasers and SLDs in PIC allows for visible-light applications but in a miniaturized platform with no moving parts (see Figure 6). Instead of relying on bulky discrete optical components, they simply use compact integrated components. These integrated devices can be useful for projection and sensing, as well as compact atomic clocks. Examples of applications of visible PICs include tunable lasers [84], flow cytometry systems [85], and laser beam steering [86], among others.

In visible PICs, SLDs can be operated either as the gain section of a tunable laser, as well as the broadband source for on-chip gyroscopes. On the other hand, single-frequency lasers, such as visible DFB lasers [88, 89] can be suitable for spectroscopy and atomic clocks. In both cases, devices with a single transverse mode are preferred for PIC applications.

The utilization of blue lasers for metal welding takes advantage of the high optical absorption of short wavelengths in metals. Blue laser diodes integrated as a laser bar or laser banks (see Figure 7) have been used for welding of steel and copper, using aggregate optical powers of up to $1 \mathrm{~kW}[90,91]$. The short 


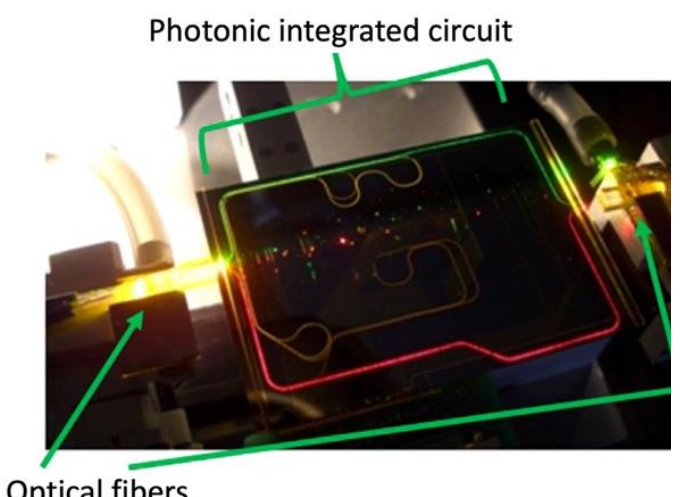

Optical fibers

Figure 6: A photonic integrated circuit (PIC) designed for visible light and the optical fibers used for light coupling and collection. Reproduced with permission from [87].

wavelength of the blue laser at $450 \mathrm{~nm}$ allows the optimization of the optical spot size and the homogeneity of the welding spot, opening a new avenue for material processing and highpower applications of visible-light laser diodes.

\section{Conclusions and perspective}

The development of semiconductor light emitters at the visible band has benefited

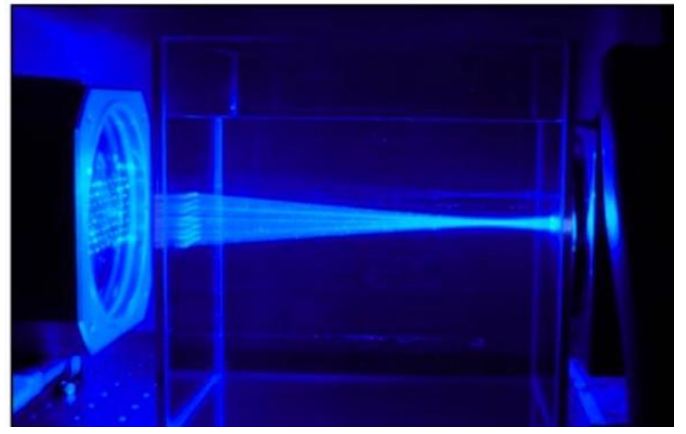

Figure 7: A bank of blue laser diodes with a total optical power of $250 \mathrm{~W}$ used for welding of metals [91]. The bank (shown on the left) consists of 64 laser diodes and a lens. The beams can be seen to be focused in the water tank.

numerous applications with scientific and economic impact. While the basic light emitters such as LEDs are the most widely spread, laser diodes and SLDs offer unique properties with value due to their degree of temporal and spatial coherency. Visible laser light beams form the basis of applications in projectors, atomic clocks, and optical communications, while SLD light enables high-resolution OCT and sensors. Table 1 provides a comparison of these semiconductor visible-light sources and

Table 1: Comparing different types of semiconductor visible-light sources

\begin{tabular}{|c|c|c|c|}
\hline Source & LEDs & SLDs & LDs \\
\hline $\begin{array}{l}\text { Temporal } \\
\text { Coherence }\end{array}$ & $\begin{array}{l}\text { Low } \\
\text { (spectral linewidth } \\
\text { of tens of } \mathrm{nm} \text { ) }\end{array}$ & $\begin{array}{l}\text { Moderately low } \\
\text { (spectral linewidth below } 10 \\
\mathrm{~nm} \text { ) }\end{array}$ & $\begin{array}{l}\text { High } \\
\text { (spectral linewidth below } 1 \\
\mathrm{~nm} \text { ) }\end{array}$ \\
\hline $\begin{array}{l}\text { Spatial } \\
\text { Coherence }\end{array}$ & Low & Relatively high & High \\
\hline Efficiency & $\begin{array}{l}\text { Suffers from } \\
\text { droop }\end{array}$ & No droop & No droop \\
\hline $\begin{array}{l}\text { Modulation } \\
\text { Bandwidth }\end{array}$ & $\begin{array}{l}10 \text { s of } \mathrm{MHz} \\
\text { (Hundreds } \mathrm{MHz} \\
\text { for micro-LED) }\end{array}$ & Hundreds of $\mathrm{MHz}$ & Beyond GHz \\
\hline $\begin{array}{l}\text { Speckle } \\
\text { Density }\end{array}$ & $\begin{array}{l}\text { Low } \\
\text { (below human } \\
\text { perception) }\end{array}$ & Moderately low & High \\
\hline $\begin{array}{l}\text { Beam } \\
\text { Divergence }\end{array}$ & High & Low & Low \\
\hline Ideal for & $\begin{array}{l}\text { Lighting, displays, } \\
\text { short-range indoor } \\
\text { communication }\end{array}$ & $\begin{array}{l}\text { Lighting, augmented and } \\
\text { virtual reality displays, } \\
\text { communication over } \\
\text { moderately long distances, } \\
\text { interference-based sensors } \\
\text { (e.g., OCT, FOG) }\end{array}$ & $\begin{array}{l}\text { Long-distance } \\
\text { communication, underwater } \\
\text { communication, high-power } \\
\text { metal welding, } \\
\text { spectroscopy, PICs, } \\
\text { horticulture, laser cooling } \\
\text { and atomic clocks }\end{array}$ \\
\hline
\end{tabular}


the advantages they offer. Both types of devices are expected to continue growing in use given their increased availability in the market and wavelength selection. Visible light offers more than just illumination. Thus, visiblelight lasers and SLDs will continue to form part of the technological environment for years to come.

\section{References}

[1] D. A. Steigerwald, J. C. Bhat, D. Collins, R. M. Fletcher, M. O. Holcomb, M. J. Ludowise, P. S. Martin, and S. L. Rudaz, "Illumination with solid state lighting technology," IEEE J. Sel. Top. Quantum Electron., vol. 8, no. 2, pp. 310-320, 2002.

[2] E. F. Schubert and J. K. Kim, "Solid-State Light Sources Getting Smart," Science, vol. 308, no. 5726, pp. 1274-1278, 2005.

[3] N. H. Jr. and S. F. Bevacqua, "Coherent (Visible) Light Emission From $\mathrm{Ga}($ As 1-xPx) Junctions," Appl. Phys. Lett., vol. 1, no. 4, pp. 82-83, 1962.

[4] R. A. Logan, H. G. White, and W. Wiegmann, "Efficient Green Electroluminescence in Nitrogen - Doped GaP $p$ - $n$ Junctions," Appl. Phys. Lett., vol. 13, no. 4, pp. 139-141, 1968.

[5] R. D. Dupuis and M. R. Krames, "History, Development, and Applications of High-Brightness Visible Light-Emitting Diodes," Journal of Lightwave Technology, vol. 26, no. 9, pp. 11541171, 2008.

[6] T. H. Maiman, "Stimulated Optical Radiation in Ruby," Nat., vol. 187, no. 4736, pp. 493-494, 1960.

[7] S. Nakamura, M. Senoh, S.-i. Nagahama, N. Iwasa, T. Yamada, T. Matsushita, H. Kiyoku, and Y. Sugimoto, "InGaN MultiQuantum-Well-Structure Laser Diodes with Cleaved Mirror Cavity Facets," Jpn. J. Appl. Phys., vol. 35, no. Part 2, No. 2B, pp. L217-L220, 1996.

[8] E. Feltin, A. Castiglia, G. Cosendey, L. Sulmoni, J.-F. Carlin, N. Grandjean, M. Rossetti, J. Dorsaz, V. Laino, M. Duelk, and C. Velez, "Broadband blue superluminescent light-emitting diodes based on GaN," Appl. Phys. Lett., vol. 95, no. 8, p. 081107, 2009.

[9] A. T. Semenov, V. R. Shidlovski, S. A. Safin, V. P. Konyaev, and M. V. Zverkov, "Superluminescent diodes for visible $(670 \mathrm{~nm})$ spectral range based on AIGalnP/GalnP heterostructures with tapered grounded absorber,"
Electronics Letters, vol. 29, no. 6, pp. 530-532, 1993.

[10] Z. I. Alferov, "Nobel Lecture: The double heterostructure concept and its applications in physics, electronics, and technology," Reviews of Modern Physics, vol. 73, no. 3, pp. 767-782, 2001.

[11] N. F. Gardner, George Craford, M. and Steranka, F.M, "Light-emitting Diodes," in digital Encyclopedia of Applied Physics: Wiley-VCH Verlag $\mathrm{GmbH}$ \& Co. KGaA (Ed.), 2004.

[12] L. A. Coldren, S. W. Corzine, and M. L. Mashanovitch, Diode Lasers and Photonic Integrated Circuits. Hoboken, United States: John Wiley \& Sons, Incorporated, 2012.

[13] Z. Khan, J.-C. Shih, R.-L. Chao, T.-L. Tsai, H.-C. Wang, G.-W. Fan, Y.-C. Lin, and J.-W. Shi, "High-brightness and highspeed vertical-cavity surface-emitting laser arrays," Optica, vol. 7, no. 4, pp. 267-275, 2020.

[14] J. Shi, J. Yan, J. Wun, J. Chen, and Y. Yang, "Oxide-Relief and Zn-Diffusion 850-nm Vertical-Cavity SurfaceEmitting Lasers With Extremely Low Energy-to-Data-Rate Ratios for 40 Gbit/s Operations," IEEE J. Sel. Top. Quantum Electron., vol. 19, no. 2, pp. 7900208-7900208, 2013

[15] H.-c. Yu, Z.-w. Zheng, Y. Mei, R.-b. Xu, J.p. Liu, H. Yang, B.-p. Zhang, T.-c. Lu, and H.-c. Kuo, "Progress and prospects of GaN-based VCSEL from near UV to green emission," Progress in Quantum Electronics, vol. 57, pp. 119, 2018.

[16] L. N. Kurbatov, S. S. Shakhidzhanov, L. V. Bystrova, V. V. Krapukhin, and S. I. Kolonenkova, "Investigation of superluminescence emitted by a gallium arsenide diode," Sov. Phys. Semicond., vol. 4, 1971.

[17] J. A. Holguin Lerma, "High-Speed GaNBased Distributed-Feedback Lasers and Optoelectronics," ed, 2020.

[18] Y. Sun, K. Zhou, Q. Sun, J. Liu, M. Feng, Z. Li, Y. Zhou, L. Zhang, D. Li, S. Zhang, M. Ikeda, S. Liu, and H. Yang, "Roomtemperature continuous-wave electrically injected InGaN-based laser directly grown on $\mathrm{Si}, "$ Nature Photonics, vol. 10, no. 9, pp. 595-599, 2016.

[19] J. Simon, V. Protasenko, C. Lian, H. Xing, and D. Jena, "Polarization-Induced Hole Doping in Wide-Band-Gap Uniaxial Semiconductor 
Heterostructures," Science, vol. 327, no. 5961, pp. 60-64, 2010.

[20] Z. Zhong, S. Lu, J. Li, W. Lin, K. Huang, S. Li, D. Cai, and J. Kang, "Design and fabrication of high power InGaN blue laser diode over $8 \mathrm{~W}$," Optics \& Laser Technology, vol. 139, p. 106985, 2021.

[21] M. Ikeda, T. Mizuno, M. Takeya, S. Goto, S. Ikeda, T. Fujimoto, Y. Ohfuji, and T. Hashizu, "High-power GaN-based semiconductor lasers," physica status solidi (c), vol. 1, no. 6, pp. 1461-1467, 2004.

[22] Y. Wang, Y. Zou, X. Ma, N. Yu, D. Deng, and K. M. Lau, "Progress on the GaNbased LEDs and LDs," in 2012 International Conference on Optoelectronics and Microelectronics, 2012, pp. 105-110.

[23] N. a. I. Ogasawara, R, "Lasers, Semiconductor," in digital Encyclopedia of Applied Physics: Wiley-VCH Verlag GmbH \& Co. KGaA (Ed.), 2004.

[24] R. Michalzik, VCSELs: Fundamentals, Technology and Applications of Vertical-Cavity Surface-Emitting Lasers. Berlin, Heidelberg: Springer Berlin Heidelberg, 2013.

[25] K. Johnson, M. Hibbs-Brenner, W. Hogan, and M. Dummer, "Advances in Red VCSEL Technology," Advances in Optical Technologies, vol. 2012, p. 569379, 2012.

[26] T. Hamaguchi, M. Tanaka, and $\mathrm{H}$. Nakajima, "A review on the latest progress of visible GaN-based VCSELs with lateral confinement by curved dielectric DBR reflector and boron ion implantation," Jpn. J. Appl. Phys., vol. 58, no. SC, p. SC0806, 2019.

[27] C. Shen, J. A. Holguin-Lerma, A. A. Alatawi, P. Zou, N. Chi, T. K. Ng, and B. S. Ooi, "Group-III-Nitride Superluminescent Diodes for Solid-State Lighting and High-Speed Visible Light Communications," IEEE J. Sel. Top. Quantum Electron., vol. 25, no. 6, pp. 1-10, 2019.

[28] A. Kafar, S. Stanczyk, D. Schiavon, T. Suski, and P. Perlin, "Review on Optimization and Current Status of (Al,In)GaN Superluminescent Diodes," ECS Journal of Solid State Science and Technology, vol. 9, no. 1, p. 015010, 2019.

[29] Q. An, J. Lin, P. Jin, and Z. Wang, "Theoretical investigation of a surfaceemitting superluminescent diode with a circular grating," Semicond. Sci. and Technol., vol. 26, no. 8, p. 085036 , 2011.

[30] B. Jentzsch, A. Gomez-Iglesias, A. Tonkikh, H. Zull, S. Kugler, and B. Witzigmann, "Surface-Emitting Micro-Mirror Superluminescent Diodes: Investigation of Tilt Accuracy Via FarField Analysis," physica status solidi (b), vol. 256, no. 8, p. 1800494, 2019.

[31] R. Cahill, P. P. Maaskant, M. Akhter, and B. Corbett, "High power surface emitting InGaN superluminescent lightemitting diodes," Appl. Phys. Lett., vol. 115, no. 17, p. 171102, 2019.

[32] K. V. Chellappan, E. Erden, and H. Urey, "Laser-based displays: a review," Appl. Opt., vol. 49, no. 25, pp. F79-F98, 2010.

[33] A. Shchegrov, A. Umbrasas, J. Watson, D. Lee, C. Amsden, W. Ha, G. Carey, V. Doan, B. Moran, A. Lewis, and A. Mooradian, "532-nm laser sources based on intracavity frequency doubling of extended-cavity surfaceemitting diode lasers," in Lasers and Applications in Science and Engineering, 2004, vol. 5332: SPIE.

[34] Y. Hirano, T. Sasagawa, T. Yanagisawa, S. Yamamoto, A. Nakamura, T. Yagi, and H. Sugiura, "Solid-state SHG green laser for laser TV," in 2009 Conference on Lasers and Electro-Optics and 2009 Conference on Quantum electronics and Laser Science Conference, 2009.

[35] S. Hallstein, G. P. Carey, R. Carico, R. Dato, J. J. Dudley, A. M. Earman, M. J. Finander, G. Giaretta, J. Green, H. J. Hofler, F. Hu, M. Jansen, C. P. Kocot, S. Lim, J. Krueger, A. Mooradian, G. Niven, Y. Okuno, F. G. Patterson, A. Tandon, and A. Umbrasas, "RGB Laser Light Sources for Projection Displays," in LEOS 2007 - IEEE Lasers and Electro-Optics Society Annual Meeting Conference Proceedings, 2007, pp. 254-255.

[36] T. Hamaguchi, Y. Hoshina, K. Hayashi, M. Tanaka, M. Ito, M. Ohara, T. Jyoukawa, N. Kobayashi, H. Watanabe, M. Yokozeki, R. Koda, and K. Yanashima, "Room-temperature continuous-wave operation of green vertical-cavity surface-emitting lasers with a curved mirror fabricated on $\{20-21\}$ semi-polar GaN," Appl. Phys. Express, vol. 13, no. 4, p. 041002, 2020.

[37] R. T. ElAfandy, J.-H. Kang, B. Li, T. K. Kim, J. S. Kwak, and J. Han, "Roomtemperature operation of c-plane GaN vertical cavity surface emitting laser on 
conductive nanoporous distributed Bragg reflector," Appl. Phys. Lett., vol. 117, no. 1, p. 011101, 2020.

[38] T. Hamaguchi, H. Nakajima, M. Tanaka, M. Ito, M. Ohara, T. Jyoukawa, N. Kobayashi, T. Matou, K. Hayashi, H. Watanabe, R. Koda, and K. Yanashima, "Sub-milliampere-threshold continuous wave operation of $\mathrm{GaN}$-based verticalcavity surface-emitting laser with lateral optical confinement by curved mirror," Appl. Phys. Express, vol. 12, no. 4, p. 044004, 2019.

[39] Y. Mei, G.-E. Weng, B.-P. Zhang, J.-P. Liu, W. Hofmann, L.-Y. Ying, J.-Y. Zhang, Z.-C. Li, H. Yang, and H.-C. Kuo, "Quantum dot vertical-cavity surfaceemitting lasers covering the 'green gap'," Light: Science \& Applications, vol. 6, no. 1, pp. e16199-e16199, 2017.

[40] P. Shukla, J. Lawrence, and Y. Zhang, "Understanding laser beam brightness: A review and new prospective in material processing," Optics \& Laser Technology, vol. 75, pp. 40-51, 2015.

[41] H. Cao, R. Chriki, S. Bittner, A. A. Friesem, and N. Davidson, "Complex lasers with controllable coherence," Nature Reviews Physics, vol. 1, no. 2, pp. 156168, 2019.

[42] L. Xu, Y. Wang, Y. Jia, and W. Zheng, "Low-coherence, high-power, highdirectional electrically driven dumbbellshaped cavity semiconductor laser at 635 nm," Opt. Lett., vol. 45, pp. 50975100, 2020.

[43] N. Primerov, J. Dahdah, S. Gloor, T. von Niederhäusern, N. Matuschek, A. Castiglia, M. Malinverni, C. Mounir, M. Rossetti, M. Duelk, and C. Vélez, "A compact red-green-blue superluminescent diode module: A novel light source for AR microdisplays," in SPIE Digital Optical Technologies, 2019, vol. 11062: SPIE.

[44] N. Primerov, J. Dahdah, S. Gloor, N. Matuschek, T. von Niederhäusern, A. Castiglia, M. Malinverni, C. Mounir, M. Rossetti, M. Duelk, and C. Vélez, "P203: Late-News Poster: Integrated Full-Color RGB Superluminescent LED Module for Micro-Displays," SID Symposium Digest of Technical Papers, vol. 50, no. 1, pp. 1731-1734, 2019.

[45] C. Martinez, V. Krotov, B. Meynard, and D. Fowler, "See-through holographic retinal projection display concept," Optica, vol. 5, pp. 1200-1209, 2018.
[46] T. Kageyama, M. Suzuki, H. Ashikaga, K. Saito, K. Yasui, S. Morino, H. Miyauchi, and $M$. Sugawara, "Visual field selfevaluation by free-focus retinalscanning laser display technology," in SPIE BiOS, 2020, vol. 11230: SPIE.

[47] J. Lin, D. Cheng, C. Yao, and Y. Wang, "Retinal projection head-mounted display," Frontiers of Optoelectronics, vol. 10, no. 1, pp. 1-8, 2017.

[48] C. Jang, K. Bang, S. Moon, J. Kim, S. Lee, and B. Lee, "Retinal 3D: augmented reality near-eye display via pupiltracked light field projection on retina," ACM Trans. Graph., vol. 36, no. 6, p. Article 190, 2017.

[49] M. Lanzagorta, "Underwater Communications," Synthesis Lectures on Communications, vol. 5, no. 2, pp. 1-129, 2012.

[50] T. Xu and L. Xu, "Chapter 1 - Introduction," in Digital Underwater Acoustic Communications, T. Xu and L. Xu, Eds.: Academic Press, 2017, pp. 1-30.

[51] H. M. Oubei, C. Shen, A. Kammoun, E. Zedini, K.-H. Park, X. Sun, G. Liu, C. H. Kang, T. K. Ng, M.-S. Alouini, and B. S. Ooi, "Light based underwater wireless communications," Jpn. J. Appl. Phys., vol. 57, no. 8S2, p. 08PA06, 2018.

[52] X. Sun, C. H. Kang, M. Kong, O. Alkhazragi, Y. Guo, M. Ouhssain, Y. Weng, B. H. Jones, T. K. Ng, and B. S. Ooi, "A Review on Practical Considerations and Solutions in Underwater Wireless Optical Communication," Journal of Lightwave Technology, vol. 38, no. 2, pp. 421-431, 2020.

[53] C. Shen, Y. Guo, H. M. Oubei, T. K. Ng, G. Liu, K.-H. Park, K.-T. Ho, M.-S. Alouini, and B. S. Ooi, "20-meter underwater wireless optical communication link with 1.5 Gbps data rate," Opt. Express, vol. 24, no. 22, pp. 25502-25509, 2016.

[54] Y. Chen, M. Kong, T. Ali, J. Wang, R. Sarwar, J. Han, C. Guo, B. Sun, N. Deng, and J. Xu, "26 m/5.5 Gbps airwater optical wireless communication based on an OFDM-modulated 520-nm laser diode," Opt. Express, vol. 25, no. 13, pp. 14760-14765, 2017.

[55] C. Yu, X. Chen, Z. Zhang, G. Song, J. Lin, and J. Xu, "Experimental verification of diffused laser beam-based optical wireless communication through air and water channels," Optics Communications, vol. 495, p. 127079 , 2021.

[56] X. Sun, M. Kong, O. Alkhazragi, C. Shen, E.-N. Ooi, X. Zhang, U. Buttner, T. K. 
$\mathrm{Ng}$, and B. S. Ooi, "Non-line-of-sight methodology for high-speed wireless optical communication in highly turbid water," Optics Communications, vol. 461, p. 125264, 2020.

[57] Y. Huang, C. Tsai, Y. Chi, D. Huang, and G. Lin, "Filtered Multicarrier OFDM Encoding on Blue Laser Diode for 14.8Gbps Seawater Transmission," Journal of Lightwave Technology, vol. 36, no. 9, pp. 1739-1745, 2018.

[58] J. Baghdady, K. Miller, K. Morgan, M. Byrd, S. Osler, R. Ragusa, W. Li, B. M. Cochenour, and E. G. Johnson, "Multigigabit/s underwater optical communication link using orbital angular momentum multiplexing," Opt. Express, vol. 24, no. 9, pp. 9794-9805, 2016.

[59] T.-C. Wu, Y.-C. Chi, H.-Y. Wang, C.-T. Tsai, and G.-R. Lin, "Blue Laser Diode Enables Underwater Communication at 12.4 Gbps," Scientific reports, vol. 7, pp. 40480-40480, 2017.

[60] X. Liu, S. Yi, R. Liu, L. Zheng, and P. Tian, "34.5 m Underwater optical wireless communication with 2.70 Gbps data rate based on a green laser with NRZOOK modulation," in 2017 14th China International Forum on Solid State Lighting: International Forum on Wide Bandgap Semiconductors China (SSLChina: IFWS), 2017, pp. 60-61.

[61] C. Li, H. Lu, W. Tsai, Z. Wang, C. Hung, C. Su, and Y. Lu, "A 5 m/25 Gbps Underwater Wireless Optical Communication System," IEEE Photonics J., vol. 10, no. 3, pp. 1-9, 2018.

[62] F. Hu, G. Li, P. Zou, J. Hu, S. Chen, Q. Liu, J. Zhang, F. Jiang, S. Wang, and N. Chi, "20.09-Gbit/s Underwater WDM-VLC Transmission based on a single $\mathrm{Si} / \mathrm{GaAs}$-substrate Multichromatic LED array chip," in Optical Fiber Communication Conference (OFC) 2020, San Diego, California, 2020, p. M3I.4: Optical Society of America.

[63] M. Chen, P. Zou, L. Zhang, and N. Chi, "Demonstration of a $2.34 \mathrm{Gbit} / \mathrm{s}$ RealTime Single Silicon-Substrate Blue LED-Based Underwater VLC System," IEEE Photonics J., vol. 12, no. 1, pp. 111, 2020.

[64] P. Tian, X. Liu, S. Yi, Y. Huang, S. Zhang, $X$. Zhou, L. Hu, L. Zheng, and R. Liu, "High-speed underwater optical wireless communication using a blue GaN-based micro-LED," Opt. Express, vol. 25, no. 2, pp. 1193-1201, 2017.
[65] H. M. Oubei, J. R. Duran, B. Janjua, H.-Y. Wang, C.-T. Tsai, Y.-C. Chi, T. K. Ng, H.-C. Kuo, J.-H. He, M.-S. Alouini, G.R. Lin, and B. S. Ooi, "4.8 Gbit/s 16QAM-OFDM transmission based on compact $450-\mathrm{nm}$ laser for underwater wireless optical communication," Opt. Express, vol. 23, no. 18, pp. 2330223309, 2015.

[66] M. Kong, W. Lv, T. Ali, R. Sarwar, C. Yu, Y. Qu, F. Qu, Z. Xu, J. Han, and J. Xu, "10-m 9.51-Gb/s RGB laser diodesbased WDM underwater wireless optical communication," Opt. Express, vol. 25, no. 17, pp. 20829-20834, 2017.

[67] H. Kaushal and G. Kaddoum, "Underwater Optical Wireless Communication," IEEE Access, vol. 4, pp. 1518-1547, 2016.

[68] L. Hildebrandt, R. Knispel, S. Stry, J. R. Sacher, and F. Schael, "Antireflectioncoated blue GaN laser diodes in an external cavity and Doppler-free indium absorption spectroscopy," Appl. Opt., vol. 42, no. 12, pp. 2110-2118, 2003.

[69] A. A. Savchenkov, J. E. Christensen, D. Hucul, W. C. Campbell, E. R. Hudson, S. Williams, and A. B. Matsko, "Application of a self-injection locked cyan laser for Barium ion cooling and spectroscopy," Scientific Reports, vol. 10, no. 1, p. 16494, 2020.

[70] V. Schkolnik, O. Fartmann, and M. Krutzik, "An extended-cavity diode laser at $497 \mathrm{~nm}$ for laser cooling and trapping of neutral strontium," Laser Physics, vol. 29, no. 3, p. 035802, 2019.

[71] K. Komori, Y. Takasu, M. Kumakura, Y. Takahashi, and T. Yabuzaki, "InjectionLocking of Blue Laser Diodes and Its Application to the Laser Cooling of Neutral Ytterbium Atoms," Jpn. J. Appl. Phys., vol. 42, no. Part 1, No. 8, pp. 5059-5062, 2003.

[72] L. Ye, L. Yi-Ge, Z. Yang, W. Qiang, W. Shao-Kai, Y. Tao, C. Jian-Ping, L. TianChu, F. Zhan-Jun, and Z. Er-Jun, "Stable Narrow Linewidth 689 nm Diode Laser for the Second Stage Cooling and Trapping of Strontium Atoms," Chinese Physics Letters, vol. 27, no. 7, p. 074208, 2010.

[73] K. R. Vogel, T. P. Dinneen, A. Gallagher, and J. L. Hall, "Narrow-line Doppler cooling of strontium to the recoil limit," IEEE Transactions on Instrumentation and Measurement, vol. 48, no. 2, pp. 618-621, 1999.

[74] A. Ooi, A. Wong, T. K. Ng, C. Marondedze, C. Gehring, and B. S. Ooi, "Growth and 
development of Arabidopsis thaliana under single-wavelength red and blue laser light," Scientific Reports, vol. 6, no. 1, p. 33885, 2016.

[75] T. Imamura, T. Inoue, and Y. Takaki, "Holographic Plant Growing System," in Digital Holography and ThreeDimensional Imaging, JeJu Island, 2017, p. W2A.2: Optical Society of America.

[76] J. Hast, L. Krehut, and R. Myllylä, "Displacement sensor based on optical feedback interferometry in a GaN laser diode," Optical Engineering, vol. 44, no. 8, p. 080504, 2005.

[77] R. A. Bergh, H. C. Lefevre, and H. J. Shaw, "All-single-mode fiber-optic gyroscope with long-term stability," Opt. Lett., vol. 6, no. 10, pp. 502-504, 1981.

[78] G. R. Goldberg, A. Boldin, S. M. L. Andersson, P. Ivanov, N. Ozaki, R. J. E. Taylor, D. T. D. Childs, K. M. Groom, K. L. Kennedy, and R. A. Hogg, "Gallium Nitride Superluminescent Light Emitting Diodes for Optical Coherence Tomography Applications," IEEE J. Sel. Top. Quantum Electron., vol. 23, no. 6, pp. 1-11, 2017.

[79] E. Andreeva, A. Anikeev, S. Il'chenko, A. Chamorovskiy, V. Shidlovski, and S. Yakubovich, "Highly efficient superluminescent diodes and SLDbased combined light sources of red spectral range for applications in biomedical imaging," in SPIE BiOS, 2018, vol. 10483: SPIE.

[80] K. Böhm, P. Marten, K. Petermann, E. Weidel, and R. Ulrich, "Low-drift fibre gyro using a superluminescent diode," Electronics Letters, vol. 17, no. 10, pp. 352-353, 1981.

[81] W. Burns, C. Chin-Lin, and R. Moeller, "Fiber-optic gyroscopes with broadband sources," Journal of Lightwave Technology, vol. 1, no. 1, pp. 98-105, 1983.

[82] R. Albertini, A. B. Villaverde, F. Aimbire, M. A. Salgado, J. M. Bjordal, L. P. Alves, E. Munin, and M. S. Costa, "Antiinflammatory effects of low-level laser therapy (LLLT) with two different red wavelengths (660 $\mathrm{nm}$ and $684 \mathrm{~nm}$ ) in carrageenan-induced rat paw edema," (in eng), J Photochem Photobiol B, vol. 89, no. 1, pp. 50-5, 2007.

[83] H. Chung, T. Dai, S. K. Sharma, Y.-Y. Huang, J. D. Carroll, and M. R. Hamblin, "The nuts and bolts of low-level laser (light) therapy," (in eng), Ann Biomed Eng, vol. 40, no. 2, pp. 516-533, 2012.
[84] A. van Rees, C. A. A. Franken, Y. Fan, D. Geskus, R. Dekker, D. H. Geuzebroek, C. Fallnich, P. J. M. van der Slot, and K.-J. Boller, "Realization of a Hybrid Integrated Diode Laser for Visible Light," in 22nd European Conference on Integrated Optics, ECIO 2020, 2020.

[85] S. Romero-García, J. Leuermann, F. Merget, J. Witzens, T. Klos, S. Koch, P. Leisching, E. Klein, D. Geuzebroek, R. Dekker, J. v. Kerkhof, J. Krieg, and M. Büscher, "Silicon nitride photonic integrated circuits for multi-color optical engines with application in flow cytometry," in 2017 IEEE Photonics Society Summer Topical Meeting Series (SUM), 2017, pp. 1-3.

[86] M. Chul Shin, A. Mohanty, K. Watson, G. R. Bhatt, C. T. Phare, S. A. Miller, M. Zadka, B. S. Lee, X. Ji, I. Datta, and M. Lipson, "Chip-scale blue light phased array," Opt. Lett., vol. 45, pp. 19341937, 2020.

[87] D. Geuzebroek, R. Dekker, E. Klein, and J. van Kerkhof, "Photonic Integrated Circuits for visible light and near infrared: Controlling transport and properties of light," Sensors and Actuators B: Chemical, vol. 223, pp. 952-956, 2016.

[88] J. A. Holguin-Lerma, M. Kong, O. Alkhazragi, X. Sun, T. Khee Ng, and B. S. Ooi, "480-nm distributed-feedback InGaN laser diode for 10.5-Gbit/s visible-light communication," Opt. Lett., vol. 45, pp. 742-745, 2020.

[89] J. A. Holguín-Lerma, T. K. Ng, and B. S. Ooi, "Narrow-line InGaN/GaN green laser diode with high-order distributedfeedback surface grating," Appl. Phys. Express, vol. 12, no. 4, p. 042007, 2019.

[90] M. Baumann, A. Balck, J. Malchus, R. Chacko, S. Marfels, U. Witte, D. Dinakaran, S. Ocylok, M. Weinbach, C. Bachert, A. Kösters, V. Krause, H. König, A. Lell, B. Stojetz, M. Ali, and U. Strauss, "1000 W blue fiber-coupled diode-laser emitting at $450 \mathrm{~nm}$," in SPIE LASE, 2019, vol. 10900: SPIE.

[91] H. Wang, Y. Kawahito, R. Yoshida, Y. Nakashima, and K. Shiokawa, "Development of a high-power blue laser (445 nm) for material processing," Opt. Lett., vol. 42, pp. 2251-2254, 2017. 